\title{
Influence of thermal treatment conditions on efficiency of PFA/ MCM-48 composite and CMK-1 carbon replica in adsorption of volatile organic compounds
}

\author{
Kamil Machowski ${ }^{1} \cdot$ Piotr Natkański $^{1} \cdot$ Anna Białas $^{1} \cdot$ Piotr Kuśtrowski $^{1}$
}

Received: 26 February 2016/ Accepted: 6 June 2016/Published online: 18 June 2016

(c) The Author(s) 2016. This article is published with open access at Springerlink.com

\begin{abstract}
The mechanism of thermal degradation of poly(furfuryl alcohol) (PFA) deposited on the MCM-48 mesoporous silica was studied. The behaviour of the thin PFA film homogeneously dispersed on the silica surface (the material with the real PFA/MCM-48 mass ratio of 0.06) was compared to that of the $3 \mathrm{D}$ structure of PFA completely filling the MCM-48 channels (the material with the real PFA/MCM-48 mass ratio of 1.02). The progress of decomposition was controlled by thermogravimetry accompanied by infrared spectroscopy providing information on the composition of gaseous products evolved during the sample degradation. The thermal treatment of PFA/ MCM-48 composites at various temperatures in the range of 523-1323 K enabled us to synthesize two series of PFAderived carbon adsorbents: (1) containing the degraded PFA film supported on the mesoporous silica and (2) CMK-1 type carbon replicas, which were obtained by leaching of $\mathrm{SiO}_{2}$ from the PFA/MCM-48 composite after the previous pyrolysis. The structural and textural properties of these materials were determined by powder X-ray diffraction, DRIFT spectroscopy and low-temperature nitrogen adsorption. Furthermore, the samples were tested as adsorbents of different volatile organic compounds (VOCs). The PFA/MCM-48 materials showed the high adsorption capacity only in the removal of polar molecules, whereas CMK-1 was effective in the elimination of linear alkanes and aromatic VOCs as well.
\end{abstract}

Piotr Kuśtrowski

kustrows@chemia.uj.edu.pl

1 Department of Chemical Technology, Faculty of Chemistry, Jagiellonian University, Ingardena 3, 30-060 Kraków, Poland
Keywords MCM-48 silica $\cdot$ Nanoreplication · Ordered mesoporous carbons · Poly(furfuryl alcohol) · Adsorption · Volatile organic compounds

\section{Introduction}

A significantly increasing concentration of volatile organic compounds (VOCs), including saturated and unsaturated aliphatic hydrocarbons, aromatic hydrocarbons as well as their derivatives, in the atmosphere is a serious environmental problem $[1,2]$. These compounds are recognized to be responsible for changes in global climate, the formation of smog, many human health problems as well as the decay of plants and animals. Therefore, useful methods of elimination of organic pollutants emitted from anthropogenic sources, such as transport, chemical and petrochemical industries or waste utilization plants, have been intensively developed. Among them, adsorption processes using suitable adsorbents - usually activated carbons (ACs) [3-5]show a high removal efficiency and many other advantages.

Lignocellulosic biomass, coal and used polymers (e.g. poly(acrylonitrile), poly(vinyl chloride), poly(ethylene terephthalate)) are widely used for the production of ACs [6-11]. However, the polymer-derived ACs usually exhibit superior structural characteristics (i.e. uniform composition, high carbon yield and low ash content) compared to the ACs prepared from the natural feedstock [12, 13]. During the production of ACs, carbonization of a raw material, performed in the temperature range of $600-900{ }^{\circ} \mathrm{C}$ under an inert atmosphere, is often followed by physical activation (for example, treatment with steam or $\mathrm{CO}_{2}$ ) and/or chemical activation (for example, reaction with $\mathrm{KOH}, \mathrm{ZnCl}_{2}$ or $\mathrm{H}_{3} \mathrm{PO}_{4}$ ), resulting in a development of 
surface area and pore volume of formed ACs as well as changes in their surface composition [14-20]. A majority of ACs is microporous solids with very narrow channels, which can cause diffusion limitations restricting an access to inner adsorption centres and making ACs unregenerable [21-24].

Ordered mesoporous carbons (OMCs) show specific surface areas comparable to those of ACs, but their significantly wider pores enable migration of large organic molecules to the inner surface $[25,26]$. OMCs are usually synthesized using: (1) soft-templating method with polymerization of a carbon precursor on micelles formed by a surfactant $[27,28]$ or (2) hard-templating method, which involves deposition of a carbon precursor inside pores of a rigid template [29-35]. The deposited carbon precursor is subsequently carbonized, and the soft or hard template is eliminated at elevated temperatures. The formed OMCs can be further activated using the same methods as mentioned above for ACs [36, 37]. The high surface area and specific surface composition cause OMCs to be active in adsorption of both small organic molecules (i.e. ethanol, toluene, $n$-hexane [38]) and larger ones (i.e. dibenzothiophene [39], aniline, fuchsine [40], orthophenanthroline, 2,2'-bipyridine [41]).

An alternative approach to obtain carbon materials containing surface functional groups characteristic of ACs is deposition of thermally activated polymer film on a high surface area support (e.g. mesoporous silica) [42]. For this purpose, polyacrylonitrile (PAN) and poly(furfuryl alcohol) (PFA) have been mainly used due to their high carbon content and thermal stability [43-45]. The highest adsorption capacity in the elimination of ketones from the gas phase was achieved for mesoporous silica decorated with small amounts of PFA pyrolysed at $250{ }^{\circ} \mathrm{C}$. It was revealed that such carbonization conditions favour the formation of surface $\gamma$-diketone moieties, which induce keto-enol tautomerization of ketone molecules and interact with them via hydrogen bonds [45].

In the presented work, we compare two series of PFAderived carbon-containing materials: (1) mesoporous silica MCM-48 decorated with a film of thermally degraded PFA and (2) CMK-1 carbon replica produced by a complete filling of mesopores in MCM-48 with PFA, subsequent carbonization and etching of $\mathrm{SiO}_{2}$ (Fig. 1). The mechanism of thermal decomposition as well as changes in structural and textural properties of materials, which occur during thermal degradation of polymeric precursor supported on the MCM-48 surface under an inert atmosphere, is studied. The CMK-1 materials, described earlier in the literature, were usually synthesized by the introduction of sucrose (as the carbon precursor) into the channels of MCM-48 mesoporous silica through impregnation in the presence of sulphuric acid catalyst followed by mild carbonization
$[33,46]$. From this point of view, the presentation of PFA as another carbon precursor, which results in a simplified route of production of CMK-1 replica with different surface composition, seems to be interesting. On the other hand, we have optimized the thermal treatment conditions in a wide temperature range from $250{ }^{\circ} \mathrm{C}$ to $1050{ }^{\circ} \mathrm{C}$ showing their influence on the adsorption efficiency of the differently pyrolysed samples in the elimination of two polar (butan-2-one and propan-2-ol) and two non-polar ( $n$ heptane and toluene) adsorbates. Such study, which evidences a possibility of using of PFA-derived OMCs with the controlled structure and surface composition, has not been presented in the literature yet.

\section{Experimental}

\section{Synthesis}

\section{$M C M-48$}

In order to obtain the MCM-48 silica support, $32 \mathrm{~mL}$ of cetyltrimethylammonium chloride $\left(25\right.$ mass $\%$ in $\mathrm{H}_{2} \mathrm{O}$, Aldrich) was mixed with $134 \mathrm{~mL}$ of distilled water, $210 \mathrm{~mL}$ of methanol (Sigma-Aldrich) and $60 \mathrm{~mL}$ of aqueous ammonia solution (25\%, Polish Chemicals Reagents) at room temperature under vigorous stirring. Then, $18 \mathrm{~mL}$ of tetraethyl orthosilicate (98\%, Aldrich) was added dropwise. The final mixture was agitated for $2 \mathrm{~h}$. Subsequently, the white precipitate was filtered, washed with distilled water and dried at $80{ }^{\circ} \mathrm{C}$ overnight. To remove the template, the dried material was calcined in air at $550{ }^{\circ} \mathrm{C}$ for $6 \mathrm{~h}$ (at a heating rate of $1{ }^{\circ} \mathrm{C} \mathrm{min}^{-1}$ ).

\section{PFA/MCM-48 composites}

The PFAx/MCM-48 composite materials, where $x=0.10$ and 1.25 indicate the intended poly(furfuryl alcohol)/ MCM-48 mass ratios, were synthesized as follows: $1.0 \mathrm{~g}$ of freshly calcined support was placed in a round-bottom flask and suspended in $100 \mathrm{~mL}$ of distilled water. Afterwards, $0.8 \mathrm{~mL}$ (for PFA0.10/MCM-48) or $10.2 \mathrm{~mL}$ (for PFA1.25/ MCM-48) of furfuryl alcohol (FA, $98 \%$, Acros Organics) was added and the mixture was stirred at room temperature for $30 \mathrm{~min}$. Subsequently, the proper volume of $\mathrm{HCl}$ (35\%, Polish Chemical Reagents), being an acid catalyst of polycondensation, was introduced at the $\mathrm{HCl} / \mathrm{FA}$ molar ratio of 6.0. The polycondensation process was performed under a reflux condenser at $100{ }^{\circ} \mathrm{C}$ for $6 \mathrm{~h}$. The obtained composite was filtered, washed with distilled water and dried at $80{ }^{\circ} \mathrm{C}$ overnight. Finally, the PFAx/MCM-48 precursors were thermally activated for $4 \mathrm{~h}$ (heating rate of $1{ }^{\circ} \mathrm{C} \min ^{-1}$ ) in a tubular furnace in flowing $\mathrm{N}_{2}$ 


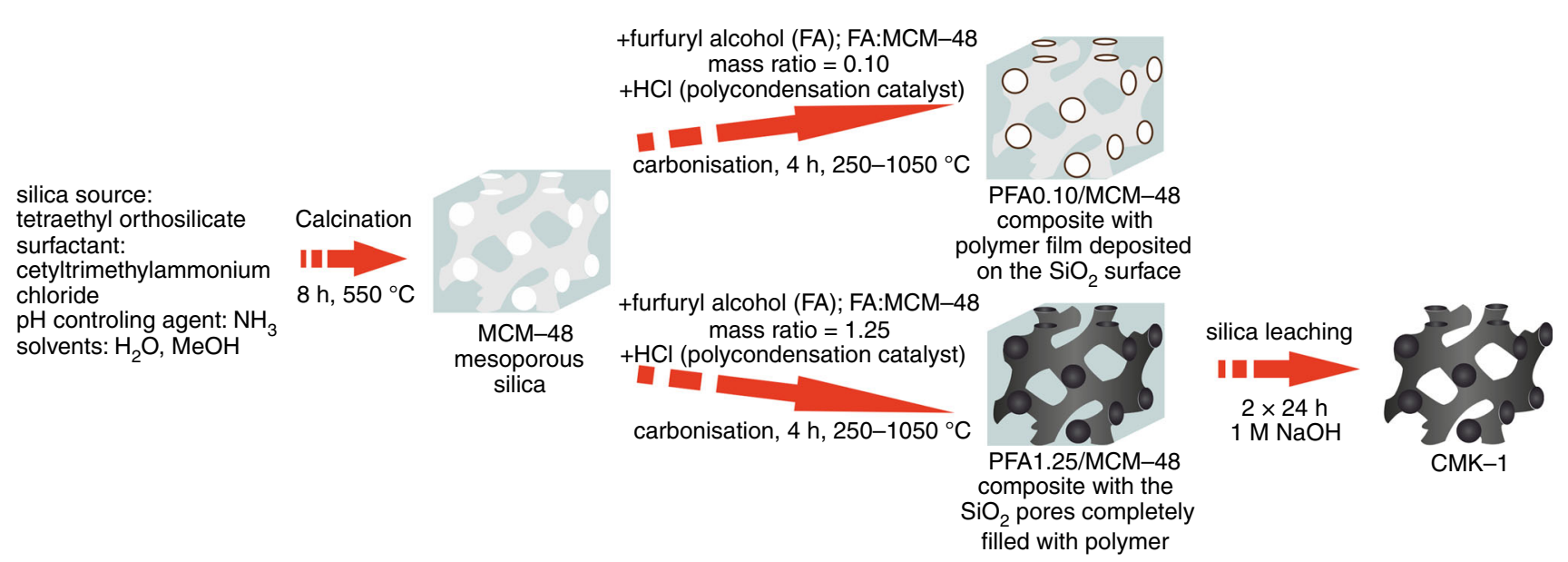

Fig. 1 Synthesis routes used to prepare the PFA-based adsorbents for the VOCs elimination

(40 $\mathrm{mL} \min ^{-1}$ ) at temperature from the range of $250-1050{ }^{\circ} \mathrm{C}$.

\section{CMK-1 carbon replica}

In order to leach silica from the thermally treated PFA1.25/ MCM-48 composites and to obtain corresponding CMK-1 carbon replicas, these samples were soaked twice in $1 \mathrm{M}$ $\mathrm{NaOH}\left(30 \mathrm{~mL} \mathrm{~g}^{-1}\right)$ for $24 \mathrm{~h}$. After that, the resulting carbon replicas were washed five times with water, once with ethanol and dried at room temperature overnight.

\section{Characterization}

The thermal decomposition of the PFAx/MCM-48 composites was studied in a TA Instruments SDT Q600 thermogravimeter equipped with a TG/FTIR interface (Thermo Scientific) connected on-line with a Nicolet 6700 FTIR spectrometer. Ca. $10 \mathrm{mg}$ of a sample was placed in a corundum crucible and outgassed in the flow of nitrogen $\left(20 \mathrm{~mL} \mathrm{~min}^{-1}\right)$ at room temperature for $1 \mathrm{~h}$. Then, the temperature was increased to $1100{ }^{\circ} \mathrm{C}$ at a heating rate of $20{ }^{\circ} \mathrm{C} \min ^{-1}$. The composition of gases evolved during the thermal treatment was analysed by collecting FTIR spectra in the wavenumber range of $650-4000 \mathrm{~cm}^{-1}$ at resolution of $4 \mathrm{~cm}^{-1}$ and time interval of $3 \mathrm{~s}$. Additional TG measurements were taken without using of the FTIR device in flowing air $\left(100 \mathrm{~mL} \mathrm{~min}{ }^{-1}\right)$ instead of $\mathrm{N}_{2}$ to determine the content of PFA in the synthesized composites.

Elemental analysis was performed using a Flash 2000 (Thermo Scientific) CHN analyser. Powder X-ray diffraction patterns (XRD) were taken using a Bruker D2 Phaser diffractometer equipped with a $\mathrm{Cu} \mathrm{K} \alpha$ radiation ( $\lambda=1.54184 \AA)$. The diffractograms were recorded in the $2 \theta$ range of $0.5^{\circ}-8.0^{\circ}$ with a step of $0.02^{\circ}$ and a count time of $1 \mathrm{~s}$ at each point. Low-temperature adsorption of nitrogen $(77 \mathrm{~K})$ was used to determine textural parameters of the studied materials. The adsorption-desorption isotherms were collected in an ASAP 2020 instrument (Micromeritics). Samples were initially outgassed at $250{ }^{\circ} \mathrm{C}$ for $12 \mathrm{~h}$. The obtained data were analysed using the BET (surface area, $S_{\mathrm{BET}}$ ) and single point (total pore volume, $V_{\text {total }}$ ) models. Moreover, the NLDFT (in the case of the MCM-48 silica and the PFAx/MCM-48 composites) and QSDFT theory (in the case of the carbon replicas) were used for the calculation of mesopore volume $\left(V_{\text {meso }}\right)$ and pore size distribution. DRIFT spectra were collected in a Nicolet 6700 (Thermo Scientific) FTIR spectrometer for samples diluted with $\mathrm{KBr}$ (4 mass \%). Two hundred scans were taken using a liquid nitrogen-cooled MCT-A detector from 650 to $4000 \mathrm{~cm}^{-1}$ at resolution of $4 \mathrm{~cm}^{-1}$.

\section{Adsorption capacity}

Ca. $100 \mathrm{mg}$ of a sample was weighted in a stainless steel reactor. The adsorbent was heated up to $250{ }^{\circ} \mathrm{C}$ in flowing $\mathrm{N}_{2}\left(40 \mathrm{~mL} \min ^{-1}\right)$ and kept at this temperature for $15 \mathrm{~min}$. Afterwards, the oven was cooled down to a temperature, at which the relative vapour pressure of selected adsorbate (butan-2-one, propan-2-ol, $n$-heptane or toluene) was 0.25 , and the flow of carrier gas $\left(\mathrm{N}_{2}, 40 \mathrm{~mL} \mathrm{~min}^{-1}\right)$, saturated with the VOCs vapour, was passed by the adsorbent bed until a flame ionization detector (FID) signal reached a plateau. After that, the system was purged with pure carrier gas in order to remove the physically adsorbed forms of VOC. Subsequently, temperature-programmed desorption (TPD) was performed by heating up to $250{ }^{\circ} \mathrm{C}$ at a rate of $15^{\circ} \mathrm{C} \min ^{-1}$ in the flow of nitrogen $\left(40 \mathrm{~mL} \mathrm{~min}{ }^{-1}\right)$. Finally, the sample was weighted in order to determine the exact mass of the run sample. The adsorption capacities were calculated from the TPD profiles after their integration. 

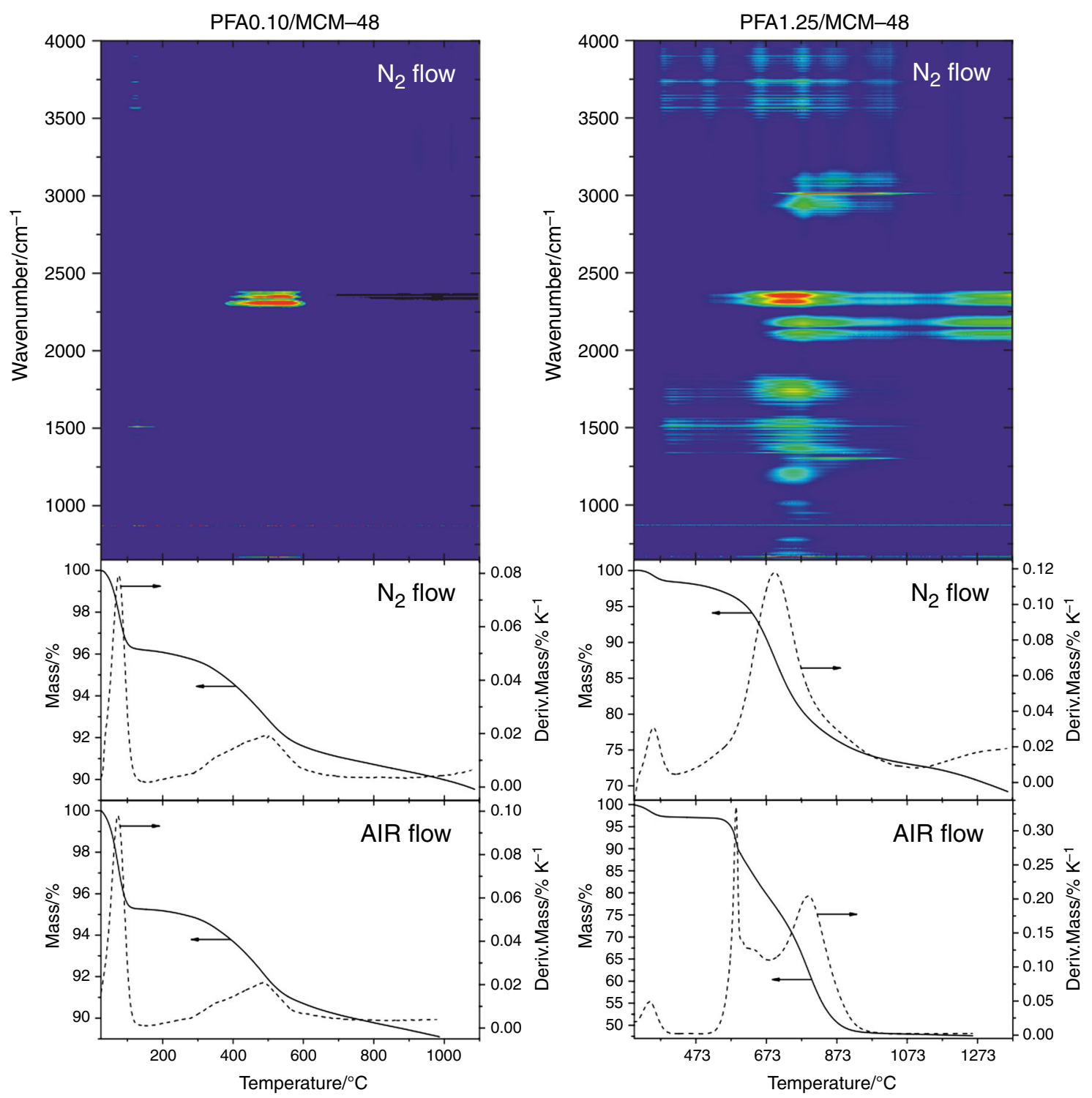

Fig. 2 TG and DTG curves as well as FTIR maps of gaseous products formed during thermal decomposition of PFA0.10/MCM-48 (left) and PFA1.25/MCM-48 (right) composites in flowing $\mathrm{N}_{2}$ and air

\section{Results and discussion}

\section{Mechanism of decomposition of PFA deposited in various amounts in pore system of MCM-48}

Thermogravimetric and elemental analyses revealed the amount of introduced PFA and the content of carbon in the synthesized PFA0.10/MCM-48 and PFA1.25/MCM-48 composites. The PFA/MCM-48 mass ratios of 0.06 and 1.02 , respectively, determined by TG, are lower than the intended values due to the incomplete deposition of furfuryl alcohol on the silica surface caused by the formation of water-soluble oligomers [44, 45]. The carbon contents in the studied materials are 1.7 and $29.4 \%$, respectively, and perfectly correspond to the calculated PFA/MCM-48 mass ratios.
The percentage mass loss observed during the TG measurements for the PFA0.10/MCM-48 sample is almost the same at both oxidizing and inert atmosphere (Fig. 2). This effect can suggest that even in flowing $\mathrm{N}_{2}$, the content of oxygen, related to its presence on the composite surface in the form of adsorbed and deposited species, is enough to combust entirely the polymer film. Figure 2 shows that the process of PFA oxidation occurs in the temperature range of $290-590{ }^{\circ} \mathrm{C}$ with the maximum at about $500{ }^{\circ} \mathrm{C}$, which corresponds to the $\mathrm{CO}_{2}$ emission manifested by the appearance of two IR bands observed at 2310 and $2350 \mathrm{~cm}^{-1}$ [47]. This finding obviously shows that the PFA film is very temperature-sensitive, and only lowtemperature activation (below $290{ }^{\circ} \mathrm{C}$ ) can protect it against any significant damage. 
(a)

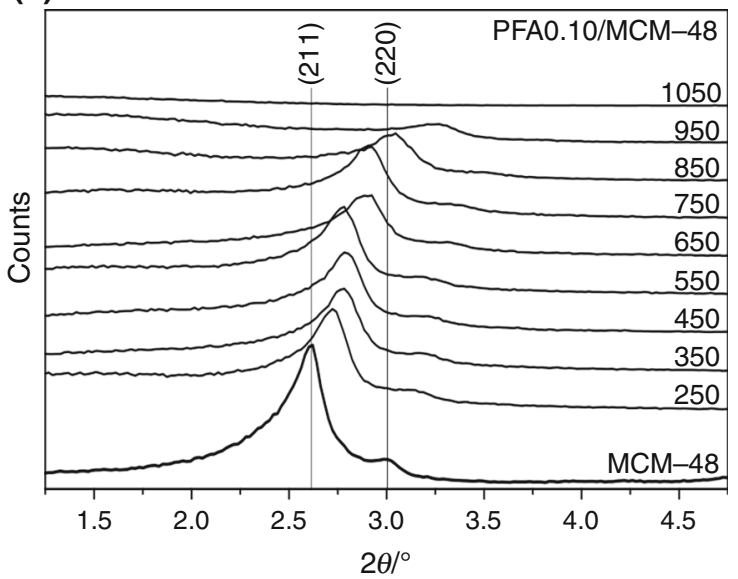

(b)

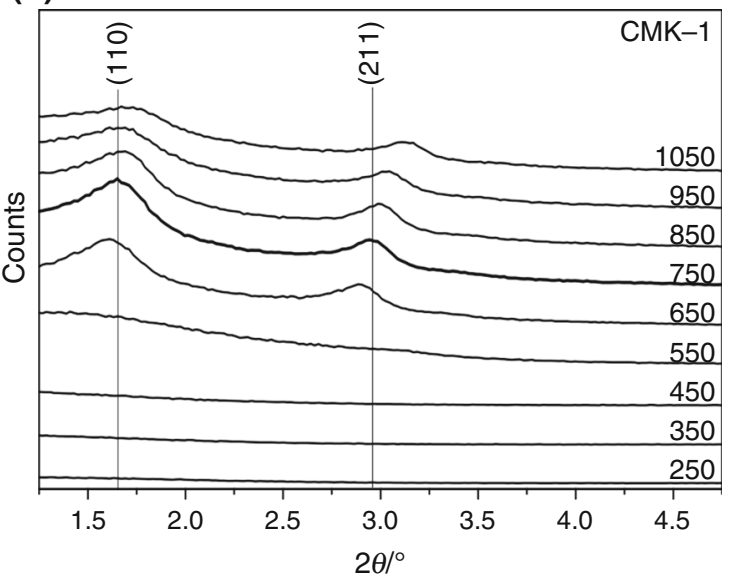

Fig. 3 XRD patterns of PFA0.10/MCM-48 composites (a) and CMK-1 carbon replicas (b) after the thermal treatment at the chosen temperature from the range of $250-1050{ }^{\circ} \mathrm{C}$

(a)

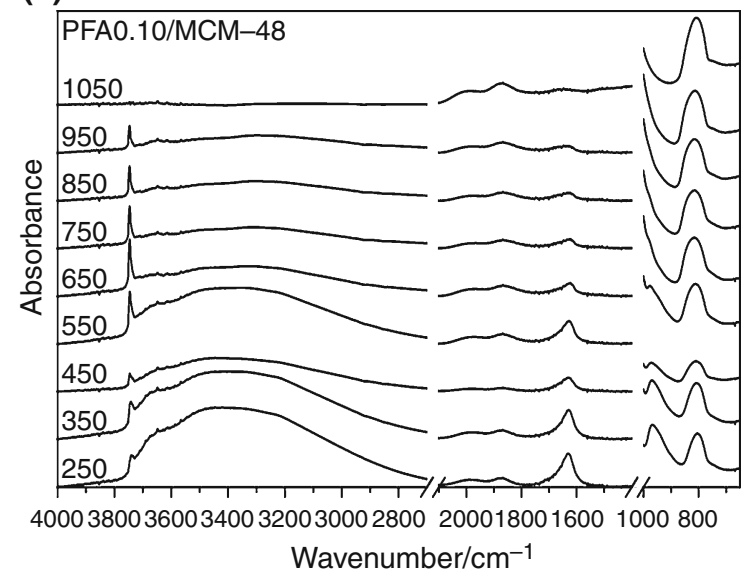

(b)

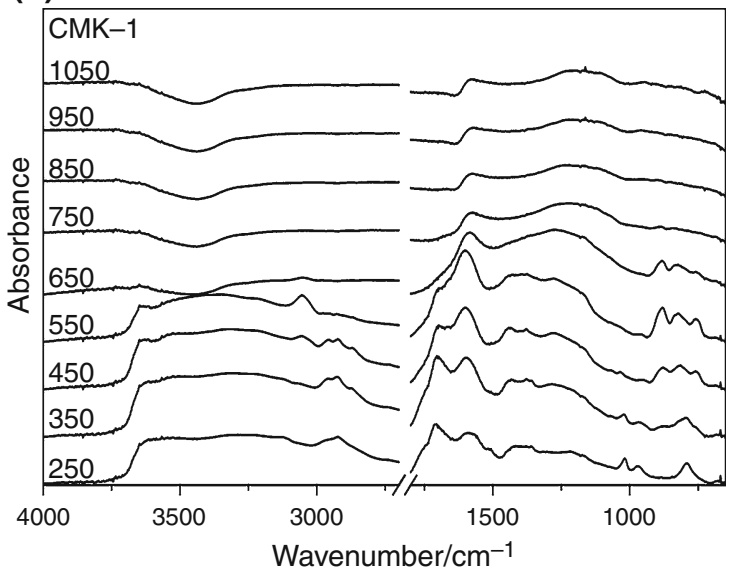

Fig. 4 DRIFT spectra of PFA0.10/MCM-48 composites (a) and CMK-1 carbon replicas (b) after the thermal treatment at the chosen temperature from the range of $250-1050{ }^{\circ} \mathrm{C}$

On the contrary, in the case of the PFA1.25/MCM-48 sample, the mass loss measured under the inert atmosphere is three times lower than in the presence of oxygen. This confirms another mechanism of thermal decomposition of PFA deposited in the $\mathrm{SiO}_{2}$ pores, which occurs in the flowing $\mathrm{N}_{2}$. The polymer degradation, with emission of $\mathrm{CO}_{2}\left(2310\right.$ and $\left.2350 \mathrm{~cm}^{-1}\right), \mathrm{CO}\left(2110\right.$ and $\left.2170 \mathrm{~cm}^{-1}\right)$ and simple carbonyl compounds (1220 and $\left.1740 \mathrm{~cm}^{-1}\right)$ detached from the ends of polymer chain [48], intensifies at about $300{ }^{\circ} \mathrm{C}$. The presence of carbonyl compounds is observed in the exhaust gases up to $650{ }^{\circ} \mathrm{C}$, while the bands attributed to $\mathrm{CO}$ and $\mathrm{CO}_{2}$ are detected even at the higher temperatures. Furthermore, the IR bands at 1304 and $3015 \mathrm{~cm}^{-1}$, which appear in the temperature range of $420-815^{\circ} \mathrm{C}$, can be assigned to the production of gaseous methane during the PFA decomposition $[49,50]$. At the temperatures higher than $815{ }^{\circ} \mathrm{C}$, the hydrogen content in the degraded polymer is probably too low to form $\mathrm{CH}_{4}$ molecules. It should therefore be assumed that above $815{ }^{\circ} \mathrm{C}$, the PFA1.25/MCM-48 material undergoes only graphitization as well as combustion by the traces of oxygen present in the purging gas.

\section{Structural and textural properties of PFA-derived adsorbents}

\section{PFA0.10/MCM-48 composites}

As previously reported [51], the structure of MCM-48 silica is thermally stable up to about $550{ }^{\circ} \mathrm{C}$. Nevertheless, 

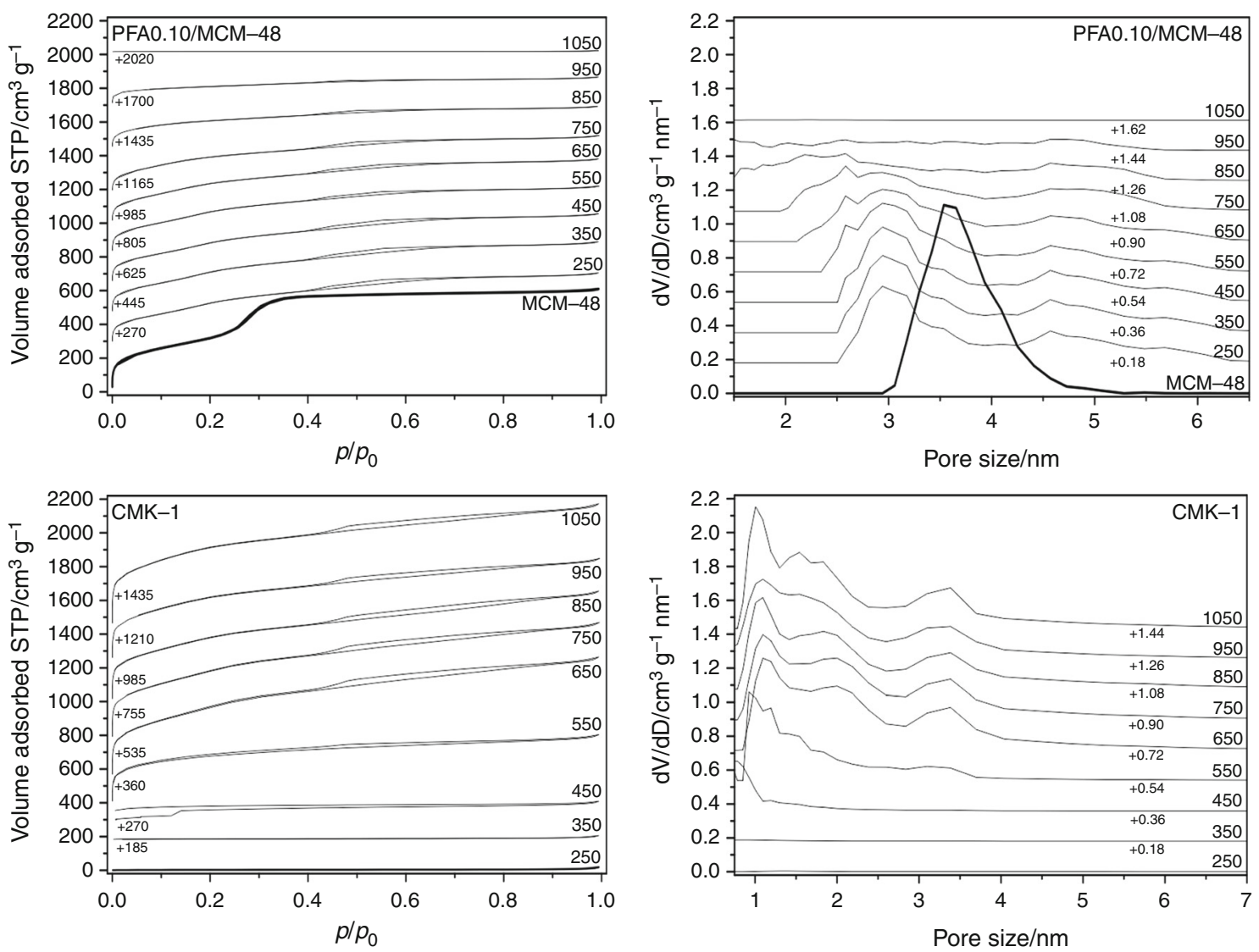

Fig. 5 Low-temperature nitrogen adsorption-desorption isotherms and pore size distributions for PFA0.10/MCM-48 composites and CMK-1 carbon replicas after the thermal treatment at the chosen temperature from the range of $250-1050{ }^{\circ} \mathrm{C}$

we studied the changes in the structural properties of PFA0.10/MCM-48 in the temperature range of 250$1050{ }^{\circ} \mathrm{C}$ using the XRD method (Fig. 3a). Both pristine MCM-48 and PFA0.10/MCM-48 composites pyrolysed at relatively low temperatures exhibit the (211) and (220) diffraction lines, which are typical of the cubic Ia $\overline{3} d$ mesostructure. The introduction of PFA followed by the thermal treatment results in the shift of (211) reflection towards higher angles. The $a_{0}$ lattice parameter calculated according to the formula of $a_{0}=6^{1 / 2} \cdot d_{211}$ changes the value from $8.3 \mathrm{~nm}$ for the pristine MCM-48 silica to $8.0 \mathrm{~nm}$ for PFA0.10/MCM- 48 activated at $250{ }^{\circ} \mathrm{C}$. A further increase in temperature causes a gradual lowering of $a_{0}$ value, finally to $7.1 \mathrm{~nm}$ for $850{ }^{\circ} \mathrm{C}$ and $6.6 \mathrm{~nm}$ for $950{ }^{\circ} \mathrm{C}$. The observed effect is explained by the shrinkage of material structure. After activation at the highest temperature, no reflection is present in the XRD pattern, suggesting the complete destruction of the porous structure of MCM-48.

The DRIFT spectra collected for the thermally activated PFA0.10/MCM-48 composite are compiled in Fig. 4a. It is clear that the increase in temperature results in the disappearance of the weak IR bands, attributed to the aliphatic -
$\mathrm{CH}$ and $-\mathrm{CH}_{2}$ stretching vibrations $(2850,2920$ and $2970 \mathrm{~cm}^{-1}$ ) in PFA deposited on the MCM-48 surface. Moreover, the decrease in the intensity of wide band at $3200-3550 \mathrm{~cm}^{-1}$, corresponding to the $\mathrm{Si}-\mathrm{OH}-\mathrm{PFA}$ interactions, connected with the increase in the intensity of sharp band at $3745 \mathrm{~cm}^{-1}$ related to the vibrations of free silanols, is observed. All these changes confirm the removal of PFA film covering the MCM-48 surface, observed by the TG analysis during the thermal treatment at the inert atmosphere (Fig. 2). It is not surprising that after heating at $1050{ }^{\circ} \mathrm{C}$, no bands characteristic of the vibrations of silanols and degraded PFA are found.

The nitrogen adsorption isotherms and pore size distribution (Fig. 5) as well as determined textural parameters (Table 1) reveal that after the deposition of PFA in the pore system of MCM-48, the BET surface area as well as mesopore and total pore volume of the silica support decrease, and simultaneously, the micropore volume increases. The pore structure of the PFA0.10/MCM- 48 composite is entirely stable up to $550{ }^{\circ} \mathrm{C}$. Above this temperature, the material begins to be transformed into an amorphous, non-porous solid. This effect is the most distinct in the case of the sample carbonized at $1050{ }^{\circ} \mathrm{C}$, 
Table 1 Textural parameters of MCM-48 mesoporous support as well as PFA0.10/MCM-48 composites and CMK-1 carbon replicas after the thermal treatment at the chosen temperature from the range of $250-1050{ }^{\circ} \mathrm{C}$

\begin{tabular}{|c|c|c|c|c|}
\hline Sample & $S_{\mathrm{BET}}^{\mathrm{a}} / \mathrm{m}^{2} \mathrm{~g}^{-1}$ & $V_{\text {micro }}^{\mathrm{b}} / \mathrm{cm}^{3} \mathrm{~g}^{-1}$ & $V_{\text {meso }}^{\mathrm{c}} / \mathrm{cm}^{3} \mathrm{~g}^{-1}$ & $V_{\text {total }}^{\mathrm{d}} / \mathrm{cm}^{3} \mathrm{~g}^{-1}$ \\
\hline MCM-48 & 1187 & 0.009 & 0.882 & 0.943 \\
\hline PFA0.10/MCM-48_523 & 999 & 0.149 & 0.627 & 0.675 \\
\hline PFA0.10/MCM-48_623 & 1010 & 0.154 & 0.635 & 0.684 \\
\hline PFA0.10/MCM-48_723 & 1026 & 0.137 & 0.616 & 0.662 \\
\hline PFA0.10/MCM-48_823 & 1044 & 0.169 & 0.593 & 0.640 \\
\hline PFA0.10/MCM-48_923 & 982 & 0.159 & 0.571 & 0.612 \\
\hline PFA0.10/MCM-48_1023 & 857 & 0.151 & 0.509 & 0.547 \\
\hline PFA0.10/MCM-48_1123 & 626 & 0.126 & 0.330 & 0.400 \\
\hline PFA0.10/MCM-48_1223 & 380 & 0.078 & 0.173 & 0.254 \\
\hline PFA0.10/MCM-48_1323 & 7 & 0.002 & 0.004 & 0.011 \\
\hline CMK-1_523 & 11 & 0.001 & 0.012 & 0.029 \\
\hline CMK-1_623 & 20 & 0.002 & 0.017 & 0.040 \\
\hline CMK-1_723 & 432 & 0.085 & 0.029 & 0.217 \\
\hline CMK-1_823 & 1124 & 0.294 & 0.190 & 0.688 \\
\hline CMK-1_923 & 1563 & 0.142 & 0.567 & 1.124 \\
\hline CMK-1_1023 & 1531 & 0.160 & 0.547 & 1.094 \\
\hline CMK-1_1123 & 1442 & 0.198 & 0.512 & 1.035 \\
\hline CMK-1_1223 & 1490 & 0.266 & 0.420 & 0.987 \\
\hline CMK-1_1323 & 1748 & 0.342 & 0.467 & 1.143 \\
\hline
\end{tabular}

which has the BET surface area and the total pore volume about 98-99\% lower than that pyrolysed at $250{ }^{\circ} \mathrm{C}$.

\section{CMK-1 carbon replicas}

The XRD patterns of CMK-1 carbon replicas carbonized at temperatures below $650{ }^{\circ} \mathrm{C}$ (Fig. 3b) reveal that these materials have no ordered mesoporous structure. Probably, so low carbonization temperatures do not provide the formation of rigid carbon backbone, which would be stable after the leaching of $\mathrm{SiO}_{2}$ template. However, the thermal treatment of PFA1.25/MCM-48 at $650{ }^{\circ} \mathrm{C}$ or higher temperature followed by the silica removal leads to the appearance of (110) and (211) diffraction lines characteristic of the cubic $I 4_{1} 32$ mesostructure. As in the case of the PFA0.10/MCM-48 composite, the increase in the activation temperature causes a slight shift of reflections to higher angles in the final CMK-1 replicas (the $a_{0}$ lattice parameter, calculated according to the formula of $a_{0}=2^{1 / 2} \cdot d_{110}$, changes its value from $7.8 \mathrm{~nm}$ for $650{ }^{\circ} \mathrm{C}$ to $7.3 \mathrm{~nm}$ for $1050{ }^{\circ} \mathrm{C}$ ). The CMK-1 sample obtained by carbonization at $750{ }^{\circ} \mathrm{C}$ shows certainly the most ordered structure.
Figure 4b shows the DRIFT spectra of the PFA1.25/ MCM-48 material after carbonization at different temperatures and the $\mathrm{SiO}_{2}$ leaching. The IR bands related to the presence of partially degraded PFA at $3400 \mathrm{~cm}^{-1}(-\mathrm{OH}$ stretching), $3115 \mathrm{~cm}^{-1}$ (-CH in aromatic rings), 2960 , 2920 and $2860 \mathrm{~cm}^{-1}$ (aliphatic $-\mathrm{CH}$ and $-\mathrm{CH}_{2}$ stretching vibrations $), \quad 1710 \mathrm{~cm}^{-1} \quad(\mathrm{C}=\mathrm{O}$ stretching vibrations $)$, $1600 \mathrm{~cm}^{-1}$ (aromatic $\mathrm{C}=\mathrm{C}$ ), $1500 \mathrm{~cm}^{-1}$ (furan ring vibrations), $1430 \mathrm{~cm}^{-1}$ (asymmetric bending $\mathrm{CH}_{2}$ vibrations), $1355 \mathrm{~cm}^{-1}$ (-CH furan ring deformation), $1020 \mathrm{~cm}^{-1}$ (asymmetric and symmetric $=\mathrm{C}-\mathrm{O}-\mathrm{C}=$ vibrations in 2-substituted furan ring) and $795 \mathrm{~cm}^{-1}$ (the vibrations of $\mathrm{C}-\mathrm{C}$ bonds in 2,5-disubstituted furan rings forming the polymer chain) are clearly observed up to $550{ }^{\circ} \mathrm{C}$. The further raising temperature results in the disappearance of these features due to the complete carbonization of PFA precursor. No new bands are distinguished in the DRIFT spectra, because the black material is characterized by high infrared absorbance.

The results of low-temperature $\mathrm{N}_{2}$ adsorption of nitrogen (Fig. 5; Table 1) confirm the evolution of CMK-1 structure with the carbonization temperature. It is obvious that the rigid framework is formed after carbonization at 
(a)

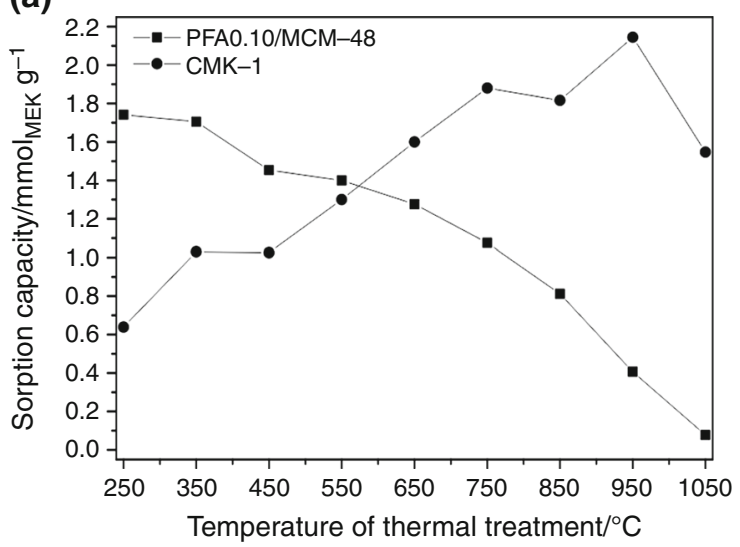

(c)

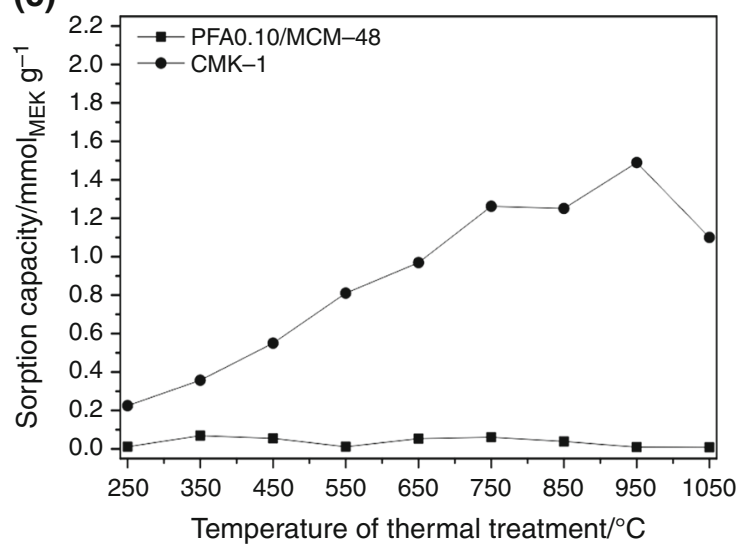

Fig. 6 Adsorption capacity (mmol of VOCs per $1 \mathrm{~g}$ of an adsorbent) of butan-2-one (a), propan-2-ol (b), $n$-heptane (c) and toluene (d) measured for PFA0.10/MCM-48 composites and CMK-1 carbon

temperature of $650{ }^{\circ} \mathrm{C}$ or even higher. This effect is manifested by a significant increase in the BET surface area as well as the mesopore volume. The CMK-1 porous structure is stable even at the highest carbonization temperature of $1050{ }^{\circ} \mathrm{C}$ when the carbon shrinkage and decomposition of surface functional groups are highly intensified. The comparison of textural properties for this sample to data collected for PFA0.10/MCM-48 pyrolysed at the same temperature reveals that the presence of PFA entirely filling the mesopores of silica protects it against structural decomposition.

\section{Adsorption capacity of PFA/MCM-48 and CMK-1 pyrolysed at different temperatures}

As shown in Fig. 6, the thermally treated PFA0.1/MCM-48 composites thermally activated at low temperatures are effective in adsorption of polar VOCs (butan-2-one and propan-2-ol). Increasing thermal treatment temperature leads to a gradual lowering of the adsorption capacity, (b)

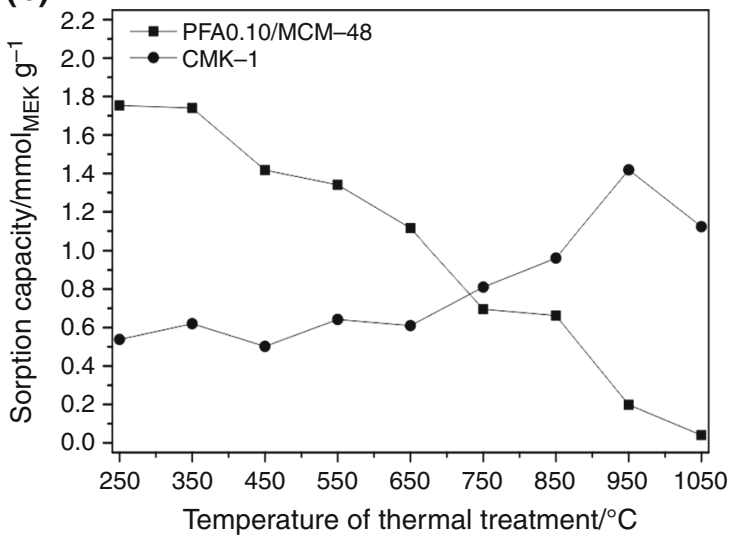

(d)

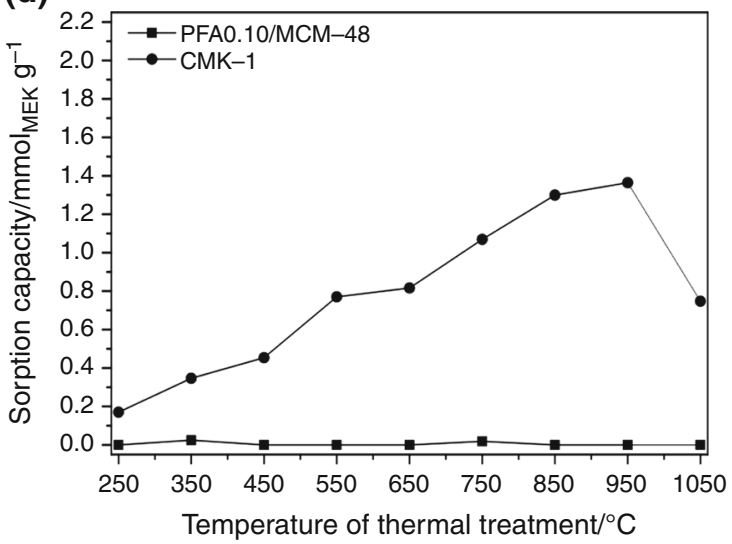

replicas after the thermal treatment at the chosen temperature from the range of $250-1050{ }^{\circ} \mathrm{C}$

which is mainly related to the progressive polymer decomposition and the elimination of surface carbonyl moieties responsible for the enhanced efficiency in the adsorption process. The high polarity of pristine silica as well as the PFA modifier causes the studied composite materials to have negligible adsorption capacity in the removal of non-polar molecules of linear alkanes and aromatic compounds ( $n$-heptane and toluene).

On the other hand, the adsorption capacity of CMK-1 carbon replicas increases with the carbonization temperature. There are at least two important reasons, which explain that fact. Firstly, the carbon materials obtained from the PFA1.25/MCM-48 composite pyrolysed at lower temperatures do not maintain the structure of template and their textural parameters are rather poor influencing the low surface concentration of adsorption centres. Secondly, the surface composition of carbon after the leaching of silica template is completely different than that of the PFA/ MCM-48 composites. The diffusion limitations during thermal treatment of MCM-48 with the pore system 
completely filled with PFA substantially change the mechanism of polymer degradation. In the consequence, the CMK-1 carbon replicas obtained from the PFA1.25/MCM48 composite carbonized at higher temperatures exhibit the affinity to both polar and non-polar adsorbates. It is most likely that the oxygen moieties co-exist with graphitic domains favouring the adsorption of various VOCs molecules. The highest adsorption capacity is achieved for the CMK-1 material carbonized at $950{ }^{\circ} \mathrm{C}$, in which the content of these species is balanced. The higher pyrolysis temperature could effectively remove the oxygen-containing groups, and too much graphitic domains appeared.

\section{Conclusions}

The mechanism of degradation of PFA deposited on the surface of MCM-48 mesoporous silica is strongly influenced by the content of polymer in the PFA/MCM-48 composite. Even in flowing nitrogen, the thin film of PFA (PFA0.10/MCM-48) is completely oxidized in the temperature range of $290-590{ }^{\circ} \mathrm{C}$ due to the presence of oxidizing species on the composite surface. On the other hand, the 3D structure of PFA located in the MCM-48 channels (PFA1.25/MCM-48) has a relatively large amount of bulk, which remains unattainable for the oxidizing agents. It allows to obtain the stable carbon structure with varying number of oxygen-containing surface functionalities and degree of graphitization.

It was observed that both PFA/MCM-48 composites and PFA-derived CMK-1 carbon replicas exhibit the cubic structure with an extremely expanded surface area and porosity. These features, controlled by the choice of conditions used during the thermal treatment, cause these materials to be very efficient adsorbents of VOCs. However, the surface composition and polarity affect the adsorption selectivity. The PFA/MCM-48 composites show the high adsorption capacity only in the removal of polar molecules, whereas CMK-1 carbons are also effective in the elimination of linear alkanes and aromatic VOCs.

\section{Acknowledgements This work was supported by the Polish National Science Centre under the grant no. 2013/11/B/ST5/01550 and by the European Regional Development Fund in the framework of the Polish Innovation Economy Operational Program (contract no. POIG.02.01.00-12-023/08), which financed the purchase of research equipment.}

Open Access This article is distributed under the terms of the Creative Commons Attribution 4.0 International License (http://crea tivecommons.org/licenses/by/4.0/), which permits unrestricted use, distribution, and reproduction in any medium, provided you give appropriate credit to the original author(s) and the source, provide a link to the Creative Commons license, and indicate if changes were made.

\section{References}

1. Chew T-L, Ahmad AL, Bhatia S. Ordered mesoporous silica (OMS) as an adsorbent and membrane for separation of carbon dioxide $\left(\mathrm{CO}_{2}\right)$. Adv Colloid Interface Sci. 2010;153:43-57.

2. Khan FI, Ghosal AK. Removal of volatile organic compounds from polluted air. J Loss Prev Process Ind. 2000;13:527-45.

3. Derbyshire F, Jagtoyen M, Andrews R, Rao A, Martín-Gullón I, Grulke E. Carbon materials in environmental applications. Marcel Decker; 2001.

4. Gallego E, Roca FJ, Perales JF, Guardino X. Experimental evaluation of VOC removal efficiency of a coconut shell activated carbon filter for indoor air quality enhancement. Build Environ. 2013;67:14-25.

5. Sidheswaran MA, Destaillats H, Sullivan DP, Cohn S, Fisk WJ. Energy efficient indoor VOC air cleaning with activated carbon fiber (ACF) filters. Build Environ. 2012;47:357-67.

6. Bansal RC, Donet BJ, Stoeckli F. Active carbon. Marcel Dekker; 1988.

7. Ryu ZY, Rong HQ, Zheng JT, Wang MZ, Zhang BJ. Microstructure and chemical analysis of PAN-based activated carbon fibers prepared by different activation methods. Carbon. 2002;40:1144-7.

8. Park SJ, Jung WY. Preparation and structural characterization of activated carbons based on polymeric resin. J Colloid Interface Sci. 2002;250:196-200.

9. San Miguel G, Fowler GD, Sollars CJ. A study of the characteristics of activated carbons produced by steam and carbon dioxide activation of waste tyre rubber. Carbon. 2003;41:1009-16.

10. Skodras G, Diamantopouiou I, Zabaniotou A, Stavropoulos G, Sakellaropoulos GP. Enhanced mercury adsorption in activated carbons from biomass materials and waste tires. Fuel Process Technol. 2007;88:749-58.

11. Long C, Lu JD, Li AM, Hu DB, Liu FQ, Zhang QX. Adsorption of naphthalene onto the carbon adsorbent from waste ion exchange resin: equilibrium and kinetic characteristics. J Hazard Mater. 2008;150:656-61.

12. Hayashi J, Yamamoto K, Horikawa T, Muroyama K, Gomes VG. Preparation and characterization of high-specific-surface-area activated carbons from $\mathrm{K}_{2} \mathrm{CO}_{3}$-treated waste polyurethane. J Colloid Interface Sci. 2005;281:437-43.

13. Vázquez-Santos MB, Castro-Muñiz A, Martínez-Alonso A, Tascón JMD. Porous texture evolution in activated carbon fibers prepared from poly ( $p$-phenylene benzobisoxazole) by carbon dioxide activation. Microporous Mesoporous Mater. 2008;116:622-6.

14. Cagnon B, Xavier P, Guillot A, Stoeckli F, Chambat G. Contributions of hemicellulose, cellulose and lignin to the mass and the porous properties of chars and steam activated carbons from various lignocellulosic precursors. Bioresource Technol. 2009;100:292-8.

15. Sun K, Jiang JC. Preparation and characterization of activated carbon from rubber-seed shell by physical activation with steam. Biomass Bioenergy. 2010;34:539-44.

16. Daud WMAW, Ali WSW, Sulaiman MZ. Effect of activation temperature on pore development in activated carbon produced from palm shell. J Chem Technol Biotechnol. 2003;78:1-5.

17. Shawabkeh RA, Aslam Z, Hussien IA. Thermochemical treatment of fly ash for synthesis of mesoporous activated carbon. J Therm Anal Calorim. 2015;122:1191-201.

18. Tsai WT, Chang CY, Lee SL. A low cost adsorbent from agricultural waste corn cob by zinc chloride activation. Bioresource Technol. 1998;64:211-7. 
19. Gupta VK, Suhas PJM. Application of low-cost adsorbents for dye removal—a review. J Environ Manage. 2009;90:2313-42.

20. Crini G. Non-conventional low-cost adsorbents for dye removal: a review. Bioresour Technol. 2006;97:1061-85.

21. Al-Othman ZA, Ali R, Naushad M. Hexavalent chromium removal from aqueous medium by activated carbon prepared from peanut shell: adsorption kinetics, equilibrium and thermodynamic studies. Chem Eng J. 2012;184:238-47.

22. Zhong ZY, Yang Q, Li XM, Luo K, Liu Y, Zeng GM. Preparation of peanut hull-based activated carbon by microwave-induced phosphoric acid activation and its application in Remazol Brilliant Blue R adsorption. Ind Crop Prod. 2012;37:178-85.

23. Liu F, Guo Z, Zheng S, Xu Z. Adsorption of tannic acid and phenol on mesoporous carbon activated by $\mathrm{CO}_{2}$. Chem Eng J. 2012;183:244-52.

24. Fu K, Yue Q, Gao B, Sun Y, Zhu L. Preparation, characterization and application of lignin-based activated carbon from black liquor lignin by steam activation. Chem Eng J. 2013;228:1074-82.

25. Zhang J, Jin L, Zhu S, Hu H. Preparation of mesoporous activated carbons from coal liquefaction residue for methane decomposition. J Nat Gas Chem. 2012;21:759-66.

26. Bedia J, Rosas JM, Rodríguez-Mirasol J, Cordero T. Pd supported on mesoporous activated carbons with high oxidation resistance as catalysts for toluene oxidation. Appl Catal B Environ. 2010;94:8-18.

27. Choma J, Jedynak K, Marszewski M, Jaroniec M. Polymertemplated mesoporous carbons synthesized in presence of nickel nanoparticles, nickel oxide nanoparticles, and nickel nitrate. Appl Surf Sci. 2012;258:3763-70.

28. Wickramaratne NP, Jaroniec M. Phenolic resin-based carbons with ultra-large mesopores prepared in the presence of poly(ethylene oxide)-poly(butylene oxide)-poly(ethylene oxide) triblock copolymer and trimethyl benzene. Carbon. 2013;51:45-51.

29. Shi ZG, Feng YQ, Xu L, Da SL, Zhang M. A template method to control the shape and porosity of carbon materials. Carbon. 2004;42:1677-82.

30. Fierro CM, Górka J, Zazo JA, Rodriguez JJ, Ludwinowicz J, Jaroniec M. Colloidal templating synthesis and adsorption characteristics of microporous-mesoporous carbons from Kraft lignin. Carbon. 2013;62:233-9.

31. Karandikar P, Patil KR, Mitra A, Kakade B, Chandwadkar AJ. Synthesis and characterization of mesoporous carbon through inexpensive mesoporous silica as template. Microporous Mesoporous Mater. 2007;98:189-99.

32. Seredych M, Bandosz TJ. Surface properties of porous carbons obtained from polystyrene-based polymers within inorganic templates: role of polymer chemistry and inorganic template pore structure. Microporous Mesoporous Mater. 2007;100:45-54.

33. Ryoo R, Joo SH, Jun S. Synthesis of highly ordered carbon molecular sieves via template-mediated structural transformation. J Phys Chem B. 1999;37:7743-6.

34. Ryoo R, Joo SH. Nanostructured carbon materials synthesized from mesoporous silica crystals by replication. Stud Surf Sci Catal. 2004; 148:241-60.

35. Jun S, Joo SH, Ryoo R, Kruk M, Jaroniec M, Liu Z, Ohsuna T, Teresaki O. Synthesis of new, nanoporous carbon with hexagonally ordered mesostructure. J Am Chem Soc. 2000;122:10712-3.
36. Górka J, Zawislak A, Choma J, Jaroniec M. KOH activation of mesoporous carbons obtained by soft-templating. Carbon. 2008;46:1159-74.

37. de Souza LKC, Wickramaratne NP, Ello AS, Costa MJF, da Costa CEF, Jaroniec M. Enhancement of $\mathrm{CO}_{2}$ adsorption on phenolic resin-based mesoporous carbons by $\mathrm{KOH}$ activation. Carbon. 2013;65:334-40.

38. Saini VK, Andrade M, Pinto ML, Carvalho AP, Pires J. How the adsorption properties get changed when going from SBA-15 to its CMK-3 carbon replica. Sep Purif Technol. 2010;75:366-76.

39. Nejad NF, Shams E, Amini MK, Bennett JC. Ordered mesoporous carbon CMK-5 as a potential sorbent for fuel desulfurization: application to the removal of dibenzothiophene and comparison with CMK-3. Microporous Mesoporous Mater. 2013;168:239-46.

40. Wu Z, Meng Y, Zhao D. Nanocasting fabrication of ordered mesoporous phenol-formaldehyde resins with various structures and their adsorption performances for basic organic compounds. Microporous Mesoporous Mater. 2010;128:165-79.

41. Anbia M, Alvand M. Fast and efficient removal of orthophenanthroline and 2,2'2,2'-bipyridine from aqueous solutions by adsorption on modified nanoporous carbon. Sci Iran C. 2012;19:1573-9.

42. Glover TG, Dunne KI, Davis RJ, LeVan MD. Carbon-silica composite adsorbent: characterization and adsorption of light gases. Microporous Mesoporous Mater. 2008;111:1-11.

43. Janus R, Kuśtrowski P, Dudek B, Piwowarska Z, Kochanowski A, Michalik M, Cool P. Removal of methyl-ethyl ketone vapour on polyacrylonitrile-derived carbon/mesoporous silica nanocomposite adsorbents. Microporous Mesoporous Mater. 2011;145:65-73.

44. Janus R, Wach A, Kuśtrowski P, Dudek B, Drozdek M, SilvestreAlbero AM, Rodríguez-Reinoso F, Cool P. Investigation on the low-temperature transformations of poly(furfuryl alcohol) deposited on MCM-41. Langmuir. 2013;29:3045-53.

45. Machowski K, Kuśtrowski P, Dudek B, Michalik M. Elimination of ketone vapors by adsorption on spherical MCM-41 and MCM48 silicas decorated with thermally activated poly(furfuryl alcohol). Mater Chem Phys. 2015;165:253-60.

46. Ryoo R, Joo SH, Kruk M, Jaroniec M. Ordered mesoporous carbons. Adv Mater. 2001;13:677-81.

47. Martin PE, Barker EF. The infrared absorption spectrum of carbon dioxide. Phys Rev. 1932;41:291-303.

48. Guigo N, Mija A, Zavaglia R, Vincent L, Sbirrazzuoli N. New insights on the thermal degradation pathways of neat poly(furfuryl alcohol) and poly(furfuryl alcohol) $/ \mathrm{SiO}_{2}$ hybrid materials. Polym Degrad Stabil. 2009;94:908-13.

49. Nelson RC, Plyler EK, Benedict WS. Absorption spectra of methane in the near infrared. $J$ Res Natl Bur Stand. 1948;41:615-21.

50. Burket CL, Rajagopalan R, Marencic AP, Dronvajjala K, Foley HC. Genesis of porosity in polyfurfuryl alcohol derived nanoporous carbon. Carbon. 2006;44:2957-63.

51. Cassiers K, Linssen T, Mathieu M, Benjelloun M, Schrijnemakers K, Van Der Voort P, Cool P, Vansant EF. A detailed study of thermal, hydrothermal, and mechanical stabilities of a wide range of surfactant assembled mesoporous silicas. Chem Mater. 2002;14:2317-24. 